\title{
eJRIEPS
}

Ejournal de la recherche sur l'intervention en éducation physique et sport

Numéro spécial 2 | 2019

Actes du congrès ARIS 2016

\section{La construction de compétences par les élèves en Education Physique : étude de la dynamique d'engagement et d'actualisation des connaissances dans l'action}

The building of competences by students in physical education: study of the dynamics of engagement and knowledge in action

Léa Gottsmann, Jean Trohel et Nathalie Gal-Petitfaux

\section{OpenEdition}

\section{Journals}

Édition électronique

URL : http://journals.openedition.org/ejrieps/931

DOI : 10.4000/ejrieps.931

ISSN : $2105-0821$

Éditeur

ELLIADD

Référence électronique

Léa Gottsmann, Jean Trohel et Nathalie Gal-Petitfaux, « La construction de compétences par les élèves en Education Physique : étude de la dynamique d'engagement et d'actualisation des connaissances dans l'action », eJRIEPS [En ligne], Numéro spécial 2 | 2019, mis en ligne le 01 juillet 2019, consulté le 01 août 2019. URL : http://journals.openedition.org/ejrieps/931 ; DOI : 10.4000/ ejrieps.931

La revue eJRIEPS est mise à disposition selon les termes de la Creative Commons Attribution 4.0 International License. 
La construction de compétences par les élèves en Education Physique : étude de la dynamique d'engagement et d'actualisation des connaissances dans l'action.

Léa Gottsmann*, Jean Trohel** et Nathalie Gal-Petitfaux**

* Université Clermont Auvergne, Laboratoire ACTé (EA 4281), Clermont-Ferrand, France.

** Université Rennes 2, Laboratoire VIPS² (EA 4636), Rennes, France.

*** Université Clermont Auvergne, Laboratoire ACTé (EA 4281), Clermont-Ferrand, France.

Résumé

L'introduction du concept de compétence dans les programmes scolaires a engendré une transformation des conceptions du savoir, passant de savoirs considérés comme universels à des savoirs d'action et d'expérience ayant une pertinence contextuelle. Nous entendons par compétence un savoir agir compétent, construit et mobilisé dans une famille de situations complexes. A travers le cadre théorique et méthodologique du cours d'action, l'objectif est d'étudier l'activité expérientielle d'une élève lors d'un cycle de badminton orienté vers la recherche du déséquilibre. II s'agit d'analyser les dynamiques de transformation des composantes de l'expérience vécue de l'élève: l'Engagement, le Representamen, les Connaissances (le Référentiel et l'Interprétant). Les résultats mettent en évidence une transformation de son activité entre les Séances 2 et 3 en passant du renvoi du volant et du respect des règles, à la recherche du déséquilibre par l'utilisation des ressources de la situation. La discussion porte sur les conditions susceptibles de contribuer à développer un agir compétent : a) les traits de familiarité entre les situations ; b) l'utilisation des artefacts ; et $c$ ) les interactions sous forme de questionnements.

Mots clés : Compétence ; Connaissance ; Savoir ; Education Physique ; Expérience.

The building of competences by students in physical education: study of the dynamics of engagement and knowledge in action.

\section{Summary}

Concept of competence in school curricula implies a transformation to knowledge, with a focus on " action knowledge " and " experiential knowledge ». Competence is knowned as 


\section{eJRIEPS spécial 2 (juillet 2019)}

a competent know-how, situated in familiar situations. Through the theoretical and methodological framework of the course of action, our objective is to analyze the experiential activity of a student during a badminton unit, in the objective to disturb the opponent. The objective is to study dynamics of the experiential components: 'Engagement', 'Representamen', and Knowledge ('Référentiel' and 'Interprétant'). The results showed transformation of those components between lessons 2 and 3. First, Jeanne is focus on the respect of rules and throw back the shuttlecock. In Lesson 3, she is focus on disturb the opponent by using rules. Discussion is about conditions which permit the construction of a competent know-how: a) the familiar structure between situations; b) the use of artefacts by the teacher; c) the questioning by the teacher to the student.

Key words : Competence ; Knowledge in action ; Knowledge ; Physical Education ; Experience.

\section{Introduction}

L'introduction d'une approche par compétences dans les textes officiels scolaires implique de repenser les savoirs du point de vue de leur fonctionnalité pratique pour agir dans des situations données. La centration sur des savoirs universels, détachés de leur contexte d'usage, cède progressivement sa place à l'acquisition de savoirs de nature: (a) contextuelle (i.e., ils sont adaptés à des situations spécifiques, tout en étant transférables au sein de classes de situations), (b) dynamique (i.e., ils sont transformables au fil des situations rencontrées), et (c) singulière (i.e., propres à l'histoire de chacun) (Masciotra \& Medzo, 2009). Cette modification de la conception des savoirs implique que ces derniers soient désormais pensés dans leurs rapports avec l'action, et envisagés comme des dispositions mobilisables pour agir dans des situations et classes de situations données (Perrenoud, 2011). C'est pourquoi dans le cadre d'un enseignement par compétences, le savoir est appréhendé comme un savoir d'action (i.e., pour servir l'action et construit à partir de l'action) et comme un savoir d'expérience (i.e., construit, mobilisé et vécu par un sujet singulier).

Dans le cadre scolaire, la rédaction des curriculums par compétences repose sur plusieurs modes d'entrées : a) celui de logiques disciplinaires qui proposent une reformulation des savoirs disciplinaires traditionnels en compétences ; b) celui des pratiques et des situations sociales ; ou c) celui des « éducations à » porteuses d'un projet social et de savoirs pour 


\section{eJRIEPS spécial 2 (juillet 2019)}

les futurs citoyens (Develay, 2015). En dépit d'une ouverture du système scolaire français aux compétences (i.e., Socle Commun de Connaissances et de Compétences, 2006 ; Socle Commun de Connaissances, de Compétences et de Culture, 2015), d'une modification de la conception du savoir comme un savoir d'action et d'expérience, le modèle des savoirs disciplinaires basé sur une hiérarchie académique et, représentant une «forteresse dispensatrice de savoirs universels » (Mons, Duru-Bellat \& Savina, 2012, p. 608) tend à rester encore majoritaire.

Pourtant, les enjeux mis en avant dans les textes officiels scolaires pour justifier l'introduction de l'approche par compétences à l'école sont clairement définis. D'abord, la compétence est considérée comme un objectif de formation pour tous les élèves afin de réduire les inégalités, de permettre à chacun d'accéder à un socle de compétences indispensables pour sa vie future et de « parvenir à davantage de justice dans la réussite scolaire » (Loi d'orientation, 2013, Article 13). L'enseignement de compétences doit aussi permettre aux élèves de construire ce qui leur permettra de «prendre part aux évolutions de la société » et de «comprendre les grands défis de l'humanité » (Socle Commun de Connaissances et de Compétences, 2006, p. 3). Face à une société en constante évolution, l'objectif est « de donner aux collégiens de nouvelles compétences adaptées au monde actuel » (Dossier « Collège : mieux apprendre pour mieux réussir », 2016, Edito). Cette formation du futur citoyen se fait particulièrement autour d'une double dimension : a) une dimension sociale cherchant « une intégration sociale et (...) une vie personnelle réussies » (Rapport Thélot, 2004, p. 24) ; et b) une dimension professionnelle où chacun doit pouvoir élever et faire évoluer son niveau de formation tout au long de sa vie.

Particulièrement en EPS, il semble que l'enseignement par compétences se développe autour d'enjeux de citoyenneté. Depuis les années 90, l'objectif est la construction de compétences chez les élèves afin qu'ils puissent les utiliser ailleurs et plus tard dans leur vie associative, sportive, artistique, mais aussi dans la gestion de leur vie physique ou encore dans leurs rapports sociaux avec les autres. Cette évolution amène de plus en plus de recherches se focalisant sur l'activité des acteurs, ce qu'ils vivent, le sens qu'ils donnent et ce qu'ils apprennent en situation. C'est donc à travers un paradigme « interprétatif » que nous allons nous intéresser aux conditions d'enseignement et d'apprentissage permettant aux élèves de construire des compétences, c'est-à-dire des savoirs d'action et d'expérience ayant une fonctionnalité contextuelle et susceptibles de répondre à de tels enjeux scolaires. 


\section{Revue de littérature}

Notre revue de littérature s'organise en trois temps. D'abord, nous exposerons une définition minimale de la compétence qui semble faire consensus au sein de la littérature et qui guidera nos réflexions. Ensuite, nous aborderons les travaux s'intéressant aux caractéristiques des situations susceptibles de contribuer au développement de compétences chez les élèves en EPS. Enfin, nous présenterons les études cherchant à analyser l'activité des élèves et de l'enseignant lors de ces situations possédant des caractéristiques favorables à la construction de compétences. Nous montrerons que, même si beaucoup d'études s'intéressent à définir ce qu'est une compétence ou ce qu'elle n'est pas, très peu d'études ont cherché à étudier le processus de construction des compétences par les élèves au cours d'une période d'apprentissage scolaire, particulièrement en EPS.

\subsection{La compétence : un agir compétent dans une famille de situations}

Un grand nombre de conceptions sont défendues dans la littérature pour définir ce qu'est une compétence, ainsi que ses conditions d'enseignement et d'apprentissage. Elles peuvent être placées sur un continuum opposant deux conceptions principales : d'un côté, la compétence comme une performance objectivable, étudiable à travers des comportements observables et mesurables; de l'autre, la compétence comme une puissance générative correspondant à une potentialité d'action propre au sujet, manifestée par la capacité de produire une infinité de conduites adaptées à des situations nouvelles (Cortesero, 2013).

La compétence est de l'ordre de l'action : qualifiée aussi d'agir compétent en situation (Masciotra \& Medzo, 2009), elle renvoie à une façon d'agir adaptée à une classe - ou une famille - de situations, ou à un champ d'expérience donné (Jonnaert, Barrette, Boufrahi \& Masciotra, 2004 ; Jonnaert, Ettayebi \& Defise, 2009 ; Meirieu, 2005 ; Nagels, 2011 ; Perrenoud, 2011 ; Rey, 2012). Considérée comme un savoir en action, mobilisable en fonction de situations données, la compétence doit être construite dans une situation particulière qui soit signifiante pour le sujet apprenant. Elle doit être étudiée dans cette même situation si l'on veut comprendre comment l'acteur la construit, la mobilise et la transforme. En effet, construire une compétence, c'est développer des "dispositions à générer des conduites adaptées face à des situations diverses " (Chauvigné \& Coulet, 2010, p. 16). Ces dispositions à agir se construisent au travers de savoirs en action et particulièrement d'un « savoir agir réfléchi » favorisant leur transfert à plusieurs situations 


\section{eJRIEPS spécial 2 (juillet 2019)}

similaires (Develay, 2015, p. 52). Dans la théorie de l'enaction, ce sont des connaissances " expérientielles » qui sont construites, structurées et/ou mobilisées dans l'expérience des individus en situation : elles sont considérées comme plus riches et plus adaptatives que des savoirs disciplinaires appris hors contexte (Masciotra, Morel \& Matthieu, 2011). II parait donc central d'étudier la compétence au travers des situations dans lesquelles elle est mise en œuvre pour comprendre comment les élèves la construisent et la transforment au cours de leur activité. Nous nous situons ici plutôt sur le pôle émancipateur de la compétence, considérée comme une disposition à agir dans des situations données.

Une autre caractéristique centrale de l'agir compétent concerne l'articulation de plusieurs types de ressources, à la fois propres à l'individu mais aussi spécifiques à la situation (Jonnaert \& al., 2009). Ces ressources sont à la fois internes (cognitives, conatives et corporelles) mais aussi externes (renvoyant aux conditions humaines et matérielles de la situation). Construire un agir compétent, ou être compétent en situation, c'est à la fois disposer de ces ressources et être capable de les mobiliser en «synergie » (Perrenoud, 2011), de façon intégrative dans un contexte (Roegiers, 2017); c'est aussi être capable de repérer des traits de similarité entre les situations et, en fonction de ces caractéristiques situationnelles, d'identifier les ressources à mobiliser pour s'y adapter. C'est l'articulation réalisée par l'individu entre les ressources de l'environnement, les savoirs codifiés mais aussi les connaissances antérieures dont il dispose, que se construisent et se transforment des connaissances pour agir, constitutives d'un agir compétent (Jonnaert \& al., 2004). Les connaissances structurant un agir compétent sont donc construites - et actualisées - en action, au fil de situations-problème particulières et représentatives d'une classe de situations : elles servent l'action et se typifient au fil de l'expérience des individus lorsqu'ils sont confrontés à ces situations-types. Aussi, pour comprendre les dynamiques de construction d'un agir compétent en EPS, il sera intéressant d'étudier la façon dont les élèves utilisent ces différentes ressources en les articulant à leurs connaissances déjà présentes, afin de construire des connaissances expérientielles leur permettant d'agir dans des situations partageant un air de famille. Cet air de famille est aussi appelé une " homologie de structure » qui invite les individus à utiliser et transférer leurs savoirs dans plusieurs situations (Rey, 1996). C'est notamment par le repérage de traits de similarité et/ou de buts similaires entre les situations que les individus parviennent à utiliser et construire des compétences (Rey, 2014).

$\mathrm{Au}$ regard de cet ensemble de caractéristiques, nous retenons donc comme définition minimale de la compétence, un «agir» compétent, constitué de connaissances 


\section{eJRIEPS spécial 2 (juillet 2019)}

expérientielles, construites en action et actualisables dans des situations possédant des airs de famille.

\subsection{Des situations de vie pour la construction de compétences}

À partir de cette définition de la compétence en termes « d'agir compétent », la question qui se pose alors est de savoir quelles sont les caractéristiques que doivent posséder les situations pour favoriser la construction et l'actualisation de cet agir compétent. À ce sujet, la littérature permet d'identifier trois caractéristiques auxquelles doivent répondre ces situations.

Tout d'abord, ces situations doivent être complexes, ou liées à la résolution d'un problème, pour permettre aux élèves de construire des connaissances garantissant une adaptation à une situation (Masciotra \& Medzo, 2009). Ces situations-problèmes doivent être signifiantes et mobilisatrices pour les élèves (Perrenoud, 1995) en étant notamment proches de situations de vie ou de situations écologiques (e.g., en EPS, proches des pratiques sociales sportives). II s'agit de situations qui sont porteuses d'incertitudes, de dilemmes et d'enjeux d'adaptation où la réponse n'est pas unique et simple (Delignières, 2009). Pour confronter les élèves à ces environnements complexes et à ces problèmes à résoudre, l'utilisation d'artefacts est un moyen qui permet de perturber l'activité des apprenants en constituant des « appels » favorisant l'apparition de nouvelles dispositions à agir (Durand, 2008). Ces propriétés des artefacts rejoignent le concept d'« espaces d'actions encouragés » (Bril, 2002), c'est à dire des espaces favorisant le déclenchement de transformations dans l'activité de l'apprenant, à partir de perturbations structurelles (Poizat, Salini \& Durand, 2013). Ces espaces complexes sont des « précurseurs d'actions et d'expériences » qui vont en encourager, en faciliter ou en valoriser (Durand, 2008, p. 108).

Une deuxième caractéristique des situations favorables à la construction et/ou l'actualisation de compétences, renvoie à la complexité des interactions sociales suscitées par ces situations. Les modalités d'interaction sociale apparaissent comme des conditions propices pour former des élèves à des compétences, en particulier aux compétences sociales (Epinoux, 2014 ; Gréhaigne, Poggi \& Zerai, 2017), indispensables à leur future vie de citoyen. Ce sont donc des « espaces d'actions et d'interactions encouragés » intégrant toutes ces caractéristiques ; qui peuvent contribuer à construire un agir compétent, à savoir : a) des objets et des instruments matériels et symboliques qui offrent des potentiels d'action aux élèves ; b) des modalités d'interaction entre les élèves favorisant la construction et/ou 


\section{eJRIEPS spécial 2 (juillet 2019)}

l'actualisation de connaissances pour agir (Saury, Adé, Gal-Petitfaux, Huet, Sève \& Trohel, 2013).

Une dernière caractéristique de ces situations concerne le fait qu'elles doivent posséder des traits de familiarité pour permettre l'identification d'éléments communs entre elles, condition nécessaire pour que soient construites des connaissances transférables et qu'elles puissent s'actualiser dans des situations de la même famille (Durand, 2008).

Ces différentes caractéristiques définissant les situations qui peuvent favoriser la construction et l'actualisation de compétences, vont nous permettre d'étudier l'activité déployée par un enseignant et les élèves en situation de construction d'un agir compétent.

\subsection{L'étude de l'agir compétent à partir d'une approche centrée « activité 》}

Un premier groupe d'études en psychologie se situent sur une approche de l'activité conçue comme une production de comportements observables et évaluables, c'est-à-dire plutôt sur le premier pôle du continuum de définitions des compétences (Cortesero, 2013). Ces études se sont intéressées à observer les compétences à travers l'analyse de comportements, jugés valides ou invalides par rapport à un modèle standardisé de la compétence. Notamment concernant les compétences sociales, des études s'accordent à définir des comportements validés socialement et évaluées à l'aide de grilles de comportements (Gendron, Royer, Bertrand \& Potvin, 2005 ; Gresham, Van \& Cook, 2006). Ces études analysent principalement l'impact d'un programme de formation aux habiletés sociales sur différentes composantes des compétences sociales (estime de soi, cognition sociale, résolution de problèmes interpersonnels). Les résultats révèlent des effets positifs immédiats pour les groupes formés. Mais l'impact de ces programmes de formation est interrogé au regard des biais possibles de désirabilité sociale, pouvant expliquer l'adoption de comportements attendus dans le cadre du programme, et qui disparaissent ensuite dans un contexte réel et social. De plus, sans un accès aux vécus et aux significations des participants lors de ces situations, il semble difficile de pouvoir identifier l'impact de ce programme sur les apprentissages réellement construits par les élèves. D'autres études dans le cadre d'activités scolaires organisées sous les principes du « Learning Together » révèlent aussi une tendance à ce que les élèves progressent davantage que ceux mis dans des groupes traditionnels (Epinoux, 2014). Mais en s'appuyant sur des tests standards à un instant t ou sur des questionnaires, ces études ne s'intéressent pas à ce que les élèves vivent en situation d'apprentissage, ni à la façon dont ils construisent et transforment leurs connaissances au cours d'une durée d'apprentissage : ce qui permettrait de rendre compte 


\section{eJRIEPS spécial 2 (juillet 2019)}

potentiellement de connaissances actualisables dans de futures situations en dehors du cadre de la classe.

Le contexte réel de classe semble essentiel pour mieux comprendre la façon dont les élèves se saisissent des éléments de l'environnement, à la fois matériels et humains. Les formes de relation entre les modalités de l'environnement et l'activité des élèves ont par exemple été analysées dans des études conduites selon le cadre du cours d'action, à travers l'analyse de l'expérience vécue des élèves. En course de durée, par exemple, il a été montré grâce à l'identification de régularités dans les préoccupations des acteurs et la caractérisation d'actions en lien avec ces préoccupations, que les éléments de l'environnement participent à structurer l'activité des élèves (Adé, Jourand \& Sève, 2010). Ces artefacts font sens pour chacun et semblent intéressants pour lancer des « appels » et créer des « potentiels d'action » chez les élèves. En musculation également, une étude sur l'utilisation par les élèves des ressources situationnelles dans un format pédagogique en ateliers révèle que des relations s'établissent et se typifient entre les actions motrices des élèves et les caractéristiques physiques des appareils (Adé, Picard \& Saury, 2013). Le réseau de ressources semble donc favoriser l'apprentissage de compétences: le fonctionnement en ateliers et les arrangements spatiaux, la nature des objets présents dans chaque atelier, et les interactions entre les élèves.

L'agir compétent se caractérise donc par la construction dans et par l'action, de connaissances expérientielles, actualisables dans des situations avec un « air de famille ». Cette activité compétente peut se développer à l'aide d'interactions entre les élèves : cellesci sont considérées comme des ressources potentielles, faisant passer d'un non-partage de connaissances à un partage partiel à partir de communications verbales entre les élèves mais aussi d'observations mutuelles (Guérin, 2011). Ce sont aussi les modes d'engagement qui sont susceptibles de contribuer à renseigner la construction de dispositions à agir, comme par exemple les trois modes typiques d'engagement qui émergent dans l'activité des élèves en classe : a) se conformer aux attentes de l'enseignant; b) construire des savoirs scolaires ; c) s'amuser et nouer des relations sociales (Guérin, op cité). Même si les deux premiers modes d'engagement sont plutôt favorables à la construction de compétences, il semble également que l'activité des élèves au cours de « jeux clandestins » ne soit pas incompatible avec les apprentissages. À travers le concept d'histoires collectives, Evin, Sève \& Saury (2013) ont étudié les processus impliqués dans la coopération entre les élèves pour favoriser leurs apprentissages. Les résultats insistent sur l'importance de favoriser le développement de significations, d'attentes et de connaissances 


\section{eJRIEPS spécial 2 (juillet 2019)}

partagées entre les élèves, en lien avec un engagement commun dans les situations. Les dynamiques de coopération peuvent donc favoriser la construction d'un agir compétent et de dispositions à agir.

Les interactions entre l'enseignant et les élèves peuvent également influencer la construction d'un agir compétent chez les élèves. En effet, les interventions de l'enseignant peuvent avoir deux sortes d'effets sur la coopération entre les élèves, et donc sur leurs apprentissages : des effets «positifs » où la coopération est facilitée entre les élèves et des effets « négatifs » qui sont nuisibles à la coopération (Evin \& al., 2013). Ces interventions influencent les dynamiques d'engagement des élèves et la coopération entre eux, et sont susceptibles d'engendrer des effets sur les dynamiques de transformation des dispositions à agir des élèves. Terré (2015) évoque ainsi trois axes de transformation des pratiques des enseignants pour aider les élèves à construire des compétences : a) aider les élèves à s'engager dans des histoires d'apprentissage ; b) aider les élèves à connecter des expériences entre elles ; c) mais aussi aider l'enseignant, en formation, à prendre en compte l'expérience des élèves. Il semble donc intéressant d'étudier l'articulation entre les attendus de l'enseignant et le vécu des élèves pour mieux comprendre comment ces derniers construisent les savoirs d'action visés par l'enseignant.

Enfin, des études ont cherché à comprendre comment les élèves réussissent à actualiser des connaissances acquises antérieurement dans d'autres situations d'apprentissage qui présentent des traits de similarité. En psychologie cognitive notamment, Richard (1990) s'est intéressé à étudier la façon dont les sujets repèrent ou non des analogies entre les situations. Les résultats montrent que les individus établissent des analogies de façon spontanée entre une situation nouvelle et des expériences passées, mais chez les individus novices, elles ne sont pas forcément adaptées aux caractéristiques spécifiques de la situation et donc n'y répondent pas de la façon la plus adéquate. Dans le cadre d'études issues du cours d'action (Theureau, 2004, 2006), l'actualisation de dispositions à agir dans plusieurs situations se fait au travers des interactions entre l'environnement et les possibilités de ressemblance entre les situations (Durand, 2008). C'est par un processus de catégorisation ou de typification des connaissances que l'agir compétent s'apprend et se développe, c'est-à-dire par la construction d'indices de typicalité qui permettent d'identifier des ressemblances et de généraliser des expériences vécues (Poizat \& al., 2013). Cette propriété est issue des travaux sur la catégorisation des objets naturels (Dubois \& Poitou, 2002 ; Rosch, 1978 ; Rosch \& Mervis, 1975) qui postulent que la cognition humaine consiste à regrouper des objets ou des événements non identiques dans des catégories. Une 


\section{eJRIEPS spécial 2 (juillet 2019)}

catégorie est un ensemble d'objets « considérés comme équivalents » par l'individu. Elle répond à deux principes : d'une part, elle est définie par l'individu sur la base d'une relation d'appartenance lui permettant de percevoir et d'interpréter si oui ou non un élément appartient à une catégorie ; d'autre part, une catégorisation n'est jamais idéalement réalisée mais s'approche graduellement d'un prototype, ce dernier étant le meilleur représentant de cette catégorie en rassemblant les conditions d'appartenance à la catégorie. Dans le cours d'action, Theureau (1992) a repris ces théories pour définir la connaissance en termes de «type » : celle-ci est construite par l'acteur à partir d'un processus de typification qui se traduit par la perception et l'interprétation de traits de similarité entre les expériences vécues par l'acteur. La typification est donc une activité cognitive par laquelle l'acteur reconnaît, en situation, certains événements singuliers en tant que phénomènes typiques, c'est-à-dire récurrents dans des contextes perçus comme similaires (Gal-Petitfaux \& Ria, 2004 ; Rosch, 1978). C'est donc ce principe de typification sur lequel nous nous appuierons pour analyser la façon dont un individu (e.g., un élève) construit une compétence. Nous nous attacherons notamment à rendre compte du processus de typification des connaissances couplé à l'action de l'élève en situation de classe. Plusieurs critères, mis à jour par la littérature, permettent de rendre compte de la typification de connaissances: a) la fréquence d'occurrences des mêmes connaissances; b) l'explicitation par l'acteur, au cours de l'entretien d'auto-confrontation, du caractère de typicité qu'il a pu repérer entre les situations qu'il a vécues ; c) l'identification de l'air de famille qui les spécifie (Durand, 2007). Cet « air de famille » renvoie aux traits les plus typiques de la catégorie de situations, reconnue par l'acteur. Les actions compétentes peuvent donc être comprises comme la traduction ou l'actualisation d'une disposition à agir en contexte, à partir d'actions typifiées au fil des environnements qu'il a rencontrés (Durand, 2008). Dans le cadre de situations de matchs en tennis de table par exemple, une étude a montré que les connaissances sont considérées comme le résultat de l'identification de ressemblances et de différences entre plusieurs expériences (Sève \& Leblanc, 2003). Ces auteurs se sont intéressés à étudier l'évolution de la cognition de joueurs au cours de situations de matchs et révèlent une alternance entre des phases d'exploration (où les préoccupations sont tournées vers la compréhension de la situation) et des phases d'exécution (où les joueurs cherchent une efficacité maximale en s'appuyant sur la familiarité des situations). Deux catégories d'action semblent alors exister en lien avec ces phases : a) des actions pragmatiques qui cherchent une efficacité immédiate, et b) des actions épistémiques qui visent la construction de nouvelles connaissances. La construction de nouvelles connaissances se fait 


\section{eJRIEPS spécial 2 (juillet 2019)}

particulièrement à partir d'observations et de généralisations issues de cas jugés similaires, en situation.

Dans le cadre scolaire, Terré (2015) a étudié le contenu des connaissances construites et mobilisées par les élèves au cours d'un cycle d'escalade par l'identification de types présents dans le Référentiel et l'Interprétant de l'acteur, en lien avec les autres composantes de l'expérience des élèves. Ces types se construisent et s'actualisent au sein "d'histoires » qui s'ouvrent, se poursuivent et se clôturent dans l'activité expérientielle de l'élève, en interaction avec son environnement, et notamment les préoccupations qui le guident. Les résultats révèlent deux conditions d'actualisation des types dans les histoires : lorsque les préoccupations sont similaires et/ou lorsque les élèves perçoivent des similitudes dans l'environnement. Cette typification des expériences corporelles antérieures par la reconnaissance d'éléments ayant des airs de famille, permet de transformer les conduites en « dispositions à agir ailleurs et plus tard » (Sève \& Gal-Petitfaux, 2015, p. 112).

Il semble donc intéressant d'étudier, au travers de l'expérience vécue des élèves en classe, la façon dont les connaissances se construisent et se transforment au cours des situations conçues par l'enseignant visant la construction d'un agir compétent, en lien avec la dynamique des engagements, des repères et des connaissances des élèves. C'est particulièrement la dynamique de transformation de ces composantes de l'expérience qui semble intéressante à étudier pour rendre compte de la construction d'un agir compétent.

\section{Problématique}

Au regard des éléments présentés en revue de littérature, nous nous intéressons donc aux dynamiques de transformation des composantes de l'expérience des élèves, à savoir l'engagement intentionnel, les repères, les connaissances actualisées et construites en action par les élèves. A partir des apprentissages visés et des situations proposées par l'enseignant, l'analyse de l'expérience vécue des élèves contribuera à mieux comprendre la façon dont les composantes de leur expérience se construisent à un instant $t$ et se modifient dans le temps. Plus précisément, nous étudierons: a) la dynamique de transformation de l'engagement des élèves au cours des situations d'apprentissage ; b) les repères pris par les élèves pour agir dans les situations, et la façon dont ils évoluent au cours du temps ; c) les connaissances qu'ils construisent et/ou actualisent pour agir, et leur dynamique de transformation au cours des différentes séquences d'apprentissage. L'analyse de la transformation des composantes de l'expérience vise à permettre d'identifier 


\section{eJRIEPS spécial 2 (juillet 2019)}

certaines conditions d'enseignement susceptibles de contribuer à la construction et à l'actualisation de connaissances structurant un agir compétent.

\section{Cadre théorique}

Pour répondre à notre problématique, nous nous appuyons sur le cadre théorique et méthodologique du cours d'action, qui propose une analyse de l'activité humaine en contexte en se focalisant sur les dimensions à la fois comportementales (observables) et expérientielles (vécues) de l'activité. Ce cadre théorique, développé par Theureau (1992, 2004, 2006) s'appuie sur plusieurs postulats (Gal-Petitfaux \& Durand, 2001) : a) l'action et la situation sont co-déterminées, c'est-à-dire que la situation est construite par l'acteur et ne prend sens pour lui que par son action dans cette situation ; b) l'action est toujours vécue par l'acteur et donc productrice de significations construites en situation, dans le présent de l'action. Ces significations produites in situ sont à la fois des émotions et des connaissances qui se construisent et/ou s'actualisent au fil des actions. Ensuite, c) les objets présents dans l'environnement sont considérés comme des artefacts cognitifs que l'acteur utilise comme affordances pour agir et penser en situation ; et d) la cognition est co-déterminée par l'acteur et la situation, c'est-à-dire distribuée entre les acteurs et la structure temporelle et spatiale de la situation.

Au regard du cours d'action, une compétence se définit comme un agir compétent : d'une part, il s'agit d'un «agir » en situation qui consiste pour l'acteur à mobiliser des connaissances « expérientielles », construites en action et pour l'action ; d'autre part, les possibilités que les individus ont d'actualiser leurs connaissances d'une situation à une autre, dépendent de leur capacité à repérer des traits de similarité entre ces situations. Au regard de ces éléments de définition, nous avons adopté le cadre du cours d'action pour conduire notre étude. Ce choix se justifie sur deux plans. Premièrement, le cours d'action est une théorie de l'activité et de l'expérience humaine fondée sur les postulats de l'action située (Suchman, 1987) et de l'enaction (Varela, 1989) : elle permet ainsi d'étudier un « agir » en situation en repérant les dimensions expérientielles (i.e., vécues) de cette activité comme élément central dans la compréhension de la construction d'un agir compétent. Deuxièmement, les outils conceptuels et méthodologiques du cours d'action nous permettent de décrire l'activité expérientielle des élèves au moyen des composantes du signe hexadique (Theureau, 2006), particulièrement quatre d'entre elles qui nous permettent de caractériser l'agir compétent des élèves : l'Engagement, le Référentiel, le Representamen et l'Interprétant. L'Engagement rend compte du faisceau de préoccupations 


\section{eJRIEPS spécial 2 (juillet 2019)}

qui animent l'acteur à chaque instant et qui émergent au fil de ses actions. Le Référentiel renvoie aux connaissances mobilisées par l'acteur : particulièrement les types, les relations entre types et les principes d'interprétation issus du cours d'action passé. Le Representamen renvoie à ce qui fait signe pour l'acteur dans la situation : il peut être perceptif, proprioceptif ou mnémonique. Enfin, l'Interprétant permet de renseigner la transformation et/ou la construction de connaissances dans l'action.

\section{Méthode}

L'étude a été menée auprès d'une classe de première dans un lycée de la banlieue rennaise, avec une élève Jeanne, et son enseignant proposant aux élèves une démarche d'apprentissage par compétences, répondant aux caractéristiques que nous avons identifiées en revue de littérature. Au cours d'un cycle de huit séances de Badminton, plusieurs données ont été recueillies : a) les documents relatifs au cycle de Badminton, au projet de classe et à l'évaluation de l'enseignant ; b) l'enregistrement audio et vidéo de l'activité de l'enseignant et de l'élève pour chaque leçon ; et c) les verbalisations issues des entretiens d'auto-confrontation avec l'enseignant, puis l'élève, à l'issue de chaque séance. Les entretiens d'auto-confrontation avaient pour objectif d'amener les participants à expliciter leurs actions et communications en classe et la façon dont ils les avaient vécues au cours des situations. Pour l'enseignant, l'objectif était surtout d'expliciter ses préoccupations et ses attendus en termes d'apprentissages visés au fil des différentes situations. Pour l'élève, des questions permettaient de renseigner les éléments du signe retenus, par exemple: «qu'est-ce que tu cherches à faire à ce momentlà ? » (Engagement) ; “à quoi fais-tu attention là ? » (Representamen) ; “ça tu le savais déjà ou tu le comprends à ce moment-là ? » (Référentiel et Interprétant).

Le traitement des données s'est ensuite déroulé en plusieurs étapes. D’abord, nous avons retranscrit et reconstitué les cours d'action de Jeanne et de l'enseignant pour chaque séance du cycle synchronisés temporellement, par la mise en correspondance des actions, des communications et des significations apportées lors de l'entretien d'auto-confrontation $(E A C)$. Nous avons ensuite renseigné les différentes composantes du signe retenues, à savoir l'Engagement (E), le Référentiel (S), le Representamen (R) et l'Interprétant (I) (Tableau I). La reconstruction du cours d'action des élèves vise à mieux comprendre les processus et les conditions favorables au développement des compétences à travers le renseignement de ces quatre composantes : l'engagement de l'élève dans la situation, ce qu'il cherche à faire ; les connaissances qu'il mobilise, qu'il construit et qu'il transforme ; ce 


\section{eJRIEPS spécial 2 (juillet 2019)}

qu'il prend en compte comme repères pour agir dans la situation : pour rendre compte d'un " air de famille » repéré par l'élève pour agir.

Enfin, nous avons étudié l'évolution de ces composantes au cours des périodes d'activité étudiées, à partir des connaissances (repérées à travers $S$ et I) en lien avec son engagement et ce qu'elle repère dans la situation, ainsi que les conditions qui en favorisent l'actualisation et la transformation.

Tableau I. Renseignement des composantes du signe.

\begin{tabular}{|l|l|l|}
\hline Composante & Nomination & Exemple \\
\hline Engagement & Verbe à l'infinitif & $\begin{array}{l}\text { "Se placer près d'une ligne pour recevoir le } \\
\text { service » }\end{array}$ \\
\hline Référentiel & $\begin{array}{l}\text { Types et relations } \\
\text { entre types }\end{array}$ & $\begin{array}{l}\text { "Il est plus facile de se mettre au fond pour } \\
\text { recevoir le service sans être en retard " }\end{array}$ \\
\hline Representamen & $\begin{array}{l}\text { Repères perceptif, } \\
\text { proprioceptif } \\
\text { mnémonique }\end{array}$ & $\begin{array}{l}\text { "Ecoute ce que lui dit l'enseignant (repère } \\
\text { perceptif) » }\end{array}$ \\
\hline Interprétant & $\begin{array}{l}\text { Validation, } \\
\text { Invalidation, } \\
\text { Construction } \\
\text { Transformation }\end{array}$ & $\begin{array}{l}\text { "C'est plus facile de se mettre sur la ligne } \\
\text { du côté que sur la ligne du fond } \\
\text { (construction) » }\end{array}$ \\
\hline
\end{tabular}

\section{Résultats}

Nous focaliserons nos résultats ici sur un axe de transformation de l'activité de Jeanne identifié lors des Séances 2 et 3 en lien avec les apprentissages visés par l'enseignant. Ces apprentissages souhaités concernent le fait d'amener les élèves à passer de l'intention d'envoyer le volant à un endroit précis recherché, vers l'intention de rechercher le déséquilibre de l'adversaire. Nous présenterons d'abord les apprentissages visés par l'enseignant ainsi que les situations mises en place pour contribuer à ces objectifs. Ensuite nous analyserons la transformation des composantes de l'expérience de Jeanne entre les Séances 2 et 3, notamment ses connaissances mobilisées et transformées, en lien avec son engagement et ce qu'elle repère dans la situation. Des focales plus précises sur des moments de transformation de son activité, permettront de cibler certaines conditions favorables au développement d'un agir compétent. 


\section{eJRIEPS spécial 2 (juillet 2019)}

\subsection{Les apprentissages visés par l'enseignant : rechercher le déséquilibre}

L'objectif de l'enseignant pour l'ensemble du cycle est de développer chez les élèves une intention de recherche de déséquilibre de l'adversaire au travers de plusieurs moyens : gêner l'adversaire, le faire se déplacer, créer le déséquilibre et conclure au bon moment. L'enseignant recoure à deux situations pédagogiques centrales tout au long du cycle, qu'il fait évoluer au moyen d'artefacts matériels et symboliques (contraintes règlementaires). La première situation correspond à des matchs sur demi-terrain, gérés au temps par l'enseignant, avec des finales gagnant/gagnant et perdant/perdant qui amènent ensuite à un mouvement des élèves en montante-descendante. La deuxième situation est basée sur la même structure, mais sur grand terrain avec arbitrage des élèves en attente.

Lors de la Séance 2, la première situation sur demi-terrain est présentée avec deux consignes : a) le serveur doit servir à un pas de la ligne du fond de terrain ; b) le receveur doit aller toucher une ligne avec le pied entre chaque frappe (sur les côtés, au fond ou au filet). Pour l'enseignant, l'objectif est d'engager les élèves dans une recherche de déséquilibre sur le déplacement : "essayez de faire le lien avec ce qu'on a vu sur les déplacements, je répète ... on travaille les déplacements, accentuez le déplacement de l'adversaire et l'espace libre » (Extrait, Séance 2, 24 minutes). La deuxième situation sur grand terrain a aussi pour consignes : a) le serveur doit servir à un ou deux pas du fond selon le niveau de jeu ; b) le receveur doit se positionner avec le pied sur une des quatre lignes (au filet, au fond ou sur les côtés) en réception de service. Là encore, l'objectif est de créer le déséquilibre : l'enseignant « là c'est moi qui ait créé l'espace libre, le déséquilibre, on va voir comment le serveur est capable d'utiliser ça » (Extrait, Séance 2, 47 minutes). Lors de la Séance 3, l'objectif est également centré sur la recherche de solutions pour augmenter le déplacement de l'adversaire au service : "essayez de répondre à cette question-là aujourd'hui : qu'est-ce qu'on peut faire pour augmenter le déplacement de l'adversaire ? »(Extrait, Séance 3, 8 minutes). La première situation sur demi-terrain a deux consignes : a) le serveur doit se placer à un ou deux pas du fond selon le niveau de jeu ; b) le receveur doit être positionné à un pas du filet. Pour l'enseignant, "là y’a déjà un double objectif (...) je crée un espace libre au service donc je vais essayer de voir s'ils sont capables de déplacer l'adversaire au service » (Extrait EAC1, Séance 3). Un espace libre correspond au fait de libérer un espace du terrain, occupé habituellement par le réceptionneur, pour encourager le serveur à utiliser cet espace et créer le déséquilibre dès le service. La deuxième situation sur grand terrain fait utiliser aux élèves des plots sur leur terrain : a) le

\footnotetext{
${ }^{1}$ EAC : Entretien d'Auto Confrontation
} 


\section{eJRIEPS spécial 2 (juillet 2019)}

serveur est à un ou deux pas devant la ligne du fond ; b) pour le receveur, trois plots sont positionnés sur son terrain (au fond, devant et sur un côté) qu'il doit toucher entre chaque frappe avec sa raquette (choix d'un plot à chaque fois). Pour l'enseignant : « je voulais qu'ils comprennent que normalement si je mets le volant à un endroit, l'adversaire il va toucher le plot le plus près, donc si l'adversaire va toucher le plot le plus près, j'ai a priori créé un espace libre à l'opposé » (Extrait EAC, Séance 3). Une troisième situation s'appuie sur la deuxième (grand terrain) mais sans les plots à aller toucher : "vous allez enlever les plots, c'est-à-dire que ça va être un jeu libre, le serveur toujours pareil à un ou deux pas du fond, vous servez où vous voulez, et le receveur ou la receveuse où elle veut » (Extrait, Séance 3, 60 minutes).

Ces situations possèdent les caractéristiques susceptibles de contribuer à la construction d'un agir compétent, telles que nous les avons repérées dans la littérature : a) elles posent un problème à l'élève au service qui doit chercher et trouver des solutions pour déséquilibrer son adversaire ; b) elles possèdent une structure et une organisation commune tout au long du cycle ; c) elles proposent différents artefacts matériels et symboliques qui viennent amplifier le problème à résoudre ou orienter l'action des élèves vers de possibles solutions ; d) elles proposent des traits culturels propres à la pratique sociale du Badminton à travers des situations de matchs.

Un des aspects de la transformation de l'activité de Jeanne concerne le passage du renvoi du volant à la recherche du déséquilibre, en lien avec les apprentissages visés par l'enseignant. Cette transformation est repérée entre les Séances 2 et 3 , au travers de plusieurs moments qui permettent d'analyser précisément la transformation des composantes de l'expérience de Jeanne, ainsi que les éléments contextuels qui favorisent ces transformations.

\subsection{Transformation de l'activité de Jeanne: vers la recherche du} déséquilibre et la construction de connaissances permettant ce déséquilibre de l'adversaire

L'activité de Jeanne entre la Séance 2 et la Séance 3 révèle d'abord une transformation notable de son engagement, qui est orienté principalement en Séance 2 vers le renvoi du volant ou vers le respect des règles du jeu, tandis qu'en Séance 3, Jeanne est orientée vers la recherche du déséquilibre de son adversaire en utilisant les différentes règles du jeu mises en place par l'enseignant. Par exemple, lors de la Séance 2 (situation sur demiterrain), Jeanne est centrée sur le fait de renvoyer le volant en face, de respecter la règle 


\section{eJRIEPS spécial 2 (juillet 2019)}

de toucher une ligne entre chaque frappe et de bien se replacer pour bien reprendre le volant (Tableau II).

Tableau II. Analyse de l'activité de Jeanne lors de la Séance 2 à 34 minutes.

\begin{tabular}{|c|c|}
\hline Séance 2-34’06 & EAC \\
\hline $\begin{array}{l}\text { Jeanne }(\mathrm{J}) \text { (en bleu clair) } \\
\text { discute avec son } \\
\text { adversaire et montre avec } \\
\text { sa raquette : “C'est la } \\
\text { ligne là au milieu » } \\
\text { Son adversaire répond: } \\
\text { «Ok» }\end{array}$ & $\begin{array}{l}\text { Chercheur }(\mathrm{C}) \text { : «Comment tu as compris la situation là ? » } \\
\mathrm{J}: \text { « Il fallait toucher les lignes sur le côté » } \\
\mathrm{C}: \text { «Et qu'est-ce que tu te dis là ? » } \\
\mathrm{J}: \text { «Bah de réussir à renvoyer les balles surtout mais pas } \\
\text { forcément à marquer des points (...) mais déjà au début je } \\
\text { pensais qu'il fallait toucher chaque ligne à chaque fois } \\
\text { chacun son tour, et donc du coup bah essayer de bien se } \\
\text { remettre au milieu pour quand le volant arrive, bien le } \\
\text { reprendre » }\end{array}$ \\
\hline & $\begin{array}{l}\text { Eléments du signe: } \\
\text { E : Renvoyer le volant et se replacer au milieu pour bien le } \\
\text { reprendre quand il arrive } \\
\text { S : Il faut toucher une ligne à chaque fois sur les côtés, } \\
\text { chacun son tour } \\
\mathbf{R}: \text { Voit les lignes du terrain (repère perceptif) } \\
\text { Se souvient des consignes de l'enseignant (repère } \\
\text { mnémonique) } \\
\text { I: Valide qu'il faut se replacer au milieu à chaque frappe pour } \\
\text { ne pas manquer le volant quand il arrive }\end{array}$ \\
\hline
\end{tabular}

Plus tard dans la séance, lors de la situation sur grand terrain, Jeanne est centrée sur le fait de se placer par rapport aux règles du jeu, c'est-à-dire la position de départ avec le pied placé sur une ligne au choix (Tableau III). 


\section{eJRIEPS spécial 2 (juillet 2019)}

Tableau III. Analyse de l'activité de Jeanne lors de la Séance 2 à 55 minutes.

\begin{tabular}{|c|c|}
\hline Séance 2 - 55’32 & EAC élève \\
\hline $\begin{array}{l}\text { Jeanne ramasse le volant et } \\
\text { le renvoie à son adversaire. } \\
\text { Elle se positionne en } \\
\text { réception de service près de } \\
\text { la ligne du fond. } \\
\text { L'enseignant vient vers elle: « }\end{array}$ & $\begin{array}{l}\text { C : «Qu'est-ce qu'il vient te dire là ? » } \\
\text { J : «Que j'étais pas obligée de me mettre tout le temps } \\
\text { dans le fond, quand c'était lui [l'adversaire] qui servait. } \\
\text { Parce que pour moi c'était plus facile mais en fait après } \\
\text { je me suis rendue compte que je me mettais plutôt au } \\
\text { niveau de la ligne bleue sur le côté » }\end{array}$ \\
\hline Alors essaie de réfléchir, toi tu & EAC enseignant \\
\hline $\begin{array}{l}\text { pars du fond, donc essaie de } \\
\text { voir comment il [l'adversaire] } \\
\text { fait est ce que toi tu as le } \\
\text { temps ou pas. Tu vois tu pars } \\
\text { de là est ce que c'est la } \\
\text { meilleure position pour partir } \\
\text { ? T'as } 4 \text { côtés, t'as le droit à } 4 \\
\text { possibilités donc réfléchis est } \\
\text { ce que c'est la meilleure } \\
\text { possibilité le meilleur endroit } \\
\text { pour partir? ». } \\
\text { Son adversaire sert et elle } \\
\text { court vers le devant du } \\
\text { terrain, mais rate le volant. }\end{array}$ & $\begin{array}{l}\text { E : «Quand j'interviens, j'essaie de les questionner sur } \\
\text { comment ils comprennent la situation mais sans trop } \\
\text { donner de solutions pour l'instant...Quand j'interviens, je } \\
\text { n'interviens jamais en donnant la solution, j'interroge } \\
\text { l'élève qu'est-ce que tu as fait, je trouve que c'est } \\
\text { important. [...] Toi de l'extérieur tu analyses la situation } \\
\text { mais quand tu dis à l'élève bah tu aurais dû faire ça bah } \\
\text { non il dit là je cherchais autre chose donc d'accord. Donc } \\
\text { c'est pour ça que c'est intéressant d'interpeller l'élève } \\
\text { par rapport à ce qu'il veut faire, et non pas forcément par } \\
\text { rapport à ce qu'il donne de l'extérieur » }\end{array}$ \\
\hline & Eléments du signe \\
\hline & $\begin{array}{l}\text { E : Se placer près d'une ligne pour recevoir le service } \\
\text { S: Il est plus facile de partir de la ligne du fond pour } \\
\text { recevoir le service } \\
\mathbf{R} \text { : Ecoute les conseils de l'enseignant (repère perceptif) } \\
\text { I : Invalide le fait que ce soit plus facile de partir de la } \\
\text { ligne du fond. } \\
\text { Comprend que c'est plus facile de se mettre sur la ligne } \\
\text { du côté (la ligne bleue) pour recevoir le service et ne pas } \\
\text { être en retard }\end{array}$ \\
\hline
\end{tabular}




\section{eJRIEPS spécial 2 (juillet 2019)}

Cet extrait révèle que Jeanne est centrée sur son placement par rapport aux lignes et aux règles mises en place par l'enseignant. Elle construit alors des connaissances liées au placement qui permet de renvoyer rapidement le service de son adversaire. Ce moment d'interaction entre l'enseignant et Jeanne permet à cette dernière d'invalider la connaissance qu'elle avait sur le fait de partir du fond pour recevoir le service, en construisant plutôt la connaissance de partir d'une ligne du côté pour être moins en retard. L'enseignant cherche d'abord à questionner Jeanne, sans lui donner d'emblée la solution : " tu vois tu pars de là, est-ce que c'est la meilleure position pour partir ? " (Extrait EAC, Séance 2). Ce moment semble faire sens pour Jeanne, qui invalide et construit une nouvelle connaissance lui permettant de partir de la ligne sur le côté pour pouvoir renvoyer le service de son adversaire plus rapidement.

Par contre, même si ce mode d'interaction permet à Jeanne de construire une nouvelle connaissance lui permettant d'agir dans la situation, il semble que pour d'autres élèves, cette modalité d'interaction sous forme de questionnement ne fasse pas toujours sens pour eux. Par exemple lors de la Séance 2, l'enseignant se déplace pour voir d'autres terrains et d'autres élèves, toujours sur le même mode de questionnement : " là tu vois avec elle, je me suis aperçu quand je lui pose des questions, bah je m'aperçois qu'elle est pas du tout dans le duel et j'essaie de lui demander mais quand tu joues aux cartes ou aux jeux de société, mais en fait elle ne fait aucune activité dans le duel ... donc là bah j'essaie de la motiver mais c'est compliqué " (EAC, Séance 2). II semble donc que ce mode de questionnement ne fasse pas sens pour tous les élèves de la classe, et ne permette pas forcément de modifier leur engagement ou leurs connaissances.

Pour revenir à l'activité de Jeanne, lors de la Séance 3, son engagement s'oriente ensuite plutôt vers la recherche du déséquilibre, en prenant des indices perceptifs sur son adversaire, notamment au service (Tableau IV). 
Tableau IV. Analyse de l'activité de Jeanne lors de la Séance 3 à 13 minutes.

\begin{tabular}{|c|c|}
\hline Séance 3-13'20 & EAC \\
\hline $\begin{array}{l}\text { Jeanne ramasse le } \\
\text { volant par terre } \\
\text { après avoir perdu } \\
\text { le point } \\
\text { Elle se place au } \\
\text { fond du terrain } \\
\text { pour servir }\end{array}$ & $\begin{array}{l}\mathrm{J}: \text { «Bah là il fallait quand on servait se mettre à un pas de la ligne } \\
\text { du fond » } \\
\mathrm{C}: \text { «Quand tu servais ? } \\
\mathrm{J}: \text { « Quand je servais, j'essayais de servir plutôt loin comme elle } \\
\text { était assez proche du filet ou sinon des fois je lançais plus proche } \\
\text { du filet mais en lui faisant croire que j'allais lancer loin avec un } \\
\text { grand geste » }\end{array}$ \\
\hline & $\begin{array}{l}\text { Eléments du signe } \\
\text { E : Servir en fonction de la position de l'adversaire en réception de } \\
\text { service } \\
\text { S : Il faut servir à l'opposé de l'endroit où se trouve son adversaire } \\
\text { (loin au fond du terrain) ou lui faire croire de lancer au fond du } \\
\text { terrain et servir proche du filet pour lui faire une feinte et le mettre } \\
\text { en retard (puisque l'adversaire s'attend à recevoir un service loin } \\
\text { au fond) } \\
\mathbf{R} \text { : Regarde si son adversaire est près ou loin du filet avant de } \\
\text { servir (repère perceptif) } \\
\text { I: Valide sa connaissance de servir à l'opposé de son adversaire }\end{array}$ \\
\hline
\end{tabular}

Cet extrait révèle les connaissances que Jeanne mobilise et valide concernant le choix au service de servir à l'opposé de l'endroit où se place son adversaire, ou bien de lui faire une feinte. Elle fait attention ici à la position de son adversaire pour choisir le service qu'elle va effectuer. Lors de la situation avec les plots sur grand terrain, Jeanne continue d'être préoccupée par la recherche du déséquilibre de son adversaire, en cherchant à augmenter les déplacements de son adversaire (Tableau V). 
Tableau V. Analyse de l'activité de Jeanne lors de la Séance 3 à 30 minutes.

\begin{tabular}{|c|c|}
\hline Séance 3-30'39 & EAC \\
\hline $\begin{array}{l}\text { Jeanne place ses plots en } \\
\text { comptant ses pas. } \\
\text { Elle va chercher un volant sur } \\
\text { le terrain d'à côté et revient en } \\
\text { courant : « c'est bon c'est bon } \\
\text { j'ai ». Elle gagne le tirage au } \\
\text { sort du service et se place au } \\
\text { service. }\end{array}$ & $\begin{array}{l}\text { C : « Donc là ta stratégie c'est quoi ? » } \\
\mathrm{J}: \text { « De jouer plutôt sur les côtés enfin gauche droite, } \\
\text { pour la faire déplacer et comme il y a des plots à toucher } \\
\text { si par exemple le volant est à gauche elle va toucher le } \\
\text { plot à gauche et du coup je vais mettre le volant à droite } \\
\text { et elle va être en retard donc la faire bouger un peu quoi } \\
\text { » }\end{array}$ \\
\hline & $\begin{array}{l}\text { Eléments du signe } \\
\text { E : Jouer sur les côtés, à gauche et à droite pour faire } \\
\text { déplacer son adversaire } \\
\text { S : Il faut déplacer son adversaire en l'obligeant à aller } \\
\text { vers tel ou tel plot } \\
\mathbf{R}: \text { Voit que son adversaire prend du retard quand elle } \\
\text { va toucher un plot (repère perceptif) } \\
\text { I : Construit qu'il faut jouer à l'inverse du plot qu'elle va } \\
\text { toucher pour la mettre en retard et la faire parcourir plus } \\
\text { de distance }\end{array}$ \\
\hline
\end{tabular}

La situation mise en place par l'enseignant cherche à amplifier le déplacement dans le jeu en obligeant un des joueurs à aller toucher un plot entre chaque frappe. Cet extrait révèle que cette situation semble faire sens pour Jeanne qui construit la connaissance de jouer à l'inverse du plot pour mettre en retard son adversaire et lui faire parcourir plus de distance. De plus, même lorsque Jeanne est en position de receveur, elle est orientée par la recherche du déséquilibre de l'adversaire, en cherchant à amener celui-ci à servir dans un certain angle (Tableau VI). 
eJRIEPS spécial 2 (juillet 2019)

Tableau VI. Analyse de l'activité de Jeanne lors de la Séance 3 à 43 minutes.

\begin{tabular}{|c|c|}
\hline Séance 3-43’02 & EAC \\
\hline $\begin{array}{l}\text { Jeanne ramasse } \\
\text { le volant et le } \\
\text { renvoie à son } \\
\text { adversaire } \\
\text { Se place du côté } \\
\text { gauche du terrain } \\
\text { pour recevoir le } \\
\text { service }\end{array}$ & $\begin{array}{l}\mathrm{C}: \text { : Et là tu changes de côté ? » } \\
\mathrm{J}: \text { « Oui j'ai changé de côté pour lui faire viser de l'autre côté, mais } \\
\text { j'ai remarqué aussi que Marine elle regardait où elle allait tirer » } \\
\mathrm{C}: \text { «Ah oui ? » } \\
\mathrm{J}: \text { « Avec ses yeux, donc quand elle faisait ça, bah je savais qu'elle } \\
\text { allait viser à gauche. La première fois je me suis dit ah bah ça doit } \\
\text { peut-être être une feinte, donc du coup je suis restée vraiment au } \\
\text { milieu et j'ai vu qu'elle faisait pareil la deuxième fois du coup je me } \\
\text { suis dit ah bah voilà » }\end{array}$ \\
\hline & $\begin{array}{l}\text { E : Se placer à gauche pour amener son adversaire à changer } \\
\text { l'angle de visée prévu } \\
\mathbf{S} \text { : Sait que son adversaire va servir à l'endroit qu'elle regarde (à } \\
\text { gauche) } \\
\mathbf{R}: \text { Voit le regard de son adversaire orienté à gauche (repère } \\
\text { perceptif) } \\
\text { I : Comprend qu'il faut qu'elle se déplace (à gauche) pour que son } \\
\text { adversaire serve à l'opposé (à droite) }\end{array}$ \\
\hline
\end{tabular}

Cet extrait révèle que Jeanne prend en compte l'endroit où regarde son adversaire pour se placer en retour de service, et pour essayer de changer son angle de service en se plaçant du côté gauche. L'engagement de Jeanne dans la recherche du déséquilibre perdure aussi Iorsqu'elle est en position de recevoir le service. Elle cherche à déséquilibrer son adversaire en prenant des repères perceptifs sur la position et le regard de son adversaire.

Lors de la dernière situation de la Séance 3, l'enseignant demande d'enlever les plots pour lancer les matchs sur grand terrain. Malgré le fait que l'objet permettant d'amplifier le déséquilibre de l'adversaire disparaisse (les plots), Jeanne continue d'être engagée vers ce déséquilibre: "là je me dis qu'il faut que je continue à faire bouger l'adversaire et à le déplacer » (Extrait EAC, Séance 3, Jeanne). 


\section{3. Éléments contextuels qui favorisent la transformation de l'activité de Jeanne}

À travers les différents moments présentés et analysés lors des Séances 2 et 3, l'activité de Jeanne se transforme de façon progressive en conformité avec les apprentissages visés par l'enseignant à propos de la recherche du déséquilibre. En effet, lors de la Séance 2, Jeanne est principalement orientée vers le renvoi du volant (Tableau II), ou vers le respect des consignes en allant toucher les lignes et en construisant des connaissances liées à ce respect des règles du jeu données par l'enseignant en situation de match (Tableau III). C'est lors de la Séance 3 que l'on voit apparaitre dans l'activité de Jeanne une recherche du déséquilibre de l'adversaire au service (Tableau IV \& V), mais aussi en retour de service par l'utilisation des règles mises en place par l'enseignant (Tableau VI). De plus, elle utilise deux principaux repères perceptifs sur son adversaire : son placement (Tableau IV \& V) et l'orientation de son regard (Tableau VI), qui lui permettent de construire et de valider des connaissances relatives à la recherche du déséquilibre en situation de match.

Deux éléments de contexte semblent intéressants à relever comme conditions favorables à la construction d'un agir compétent chez les élèves : les caractéristiques des situations mises en place par l'enseignant, et le format des interactions entre l'enseignant et Jeanne. Tout d'abord, les caractéristiques des situations mises en place par l'enseignant semblent intéressantes pour amplifier la recherche d'espaces libres afin d'amener Jeanne à transformer son activité vers la recherche du déséquilibre. Les différents artefacts utilisés (règles pour le serveur, le receveur, les plots et lignes à aller toucher) servent à rendre plus saillant le déséquilibre pour que les élèves s'engagent dans cette recherche du déséquilibre et développent des connaissances liées à cet objectif. De plus, ces situations posent un problème aux élèves : ils doivent trouver des moyens de déséquilibrer l'adversaire au service, mais ils doivent aussi de ne pas prendre trop de retard lorsqu'ils sont en position de toucher les plots ou les lignes (position de receveur).

Ensuite, l'enseignant fait le choix de questionner les élèves lorsqu'ils passent entre les terrains (Tableau III). Son objectif est d'interroger ce que fait l'élève, sans forcément lui donner la solution, mais pour essayer de l'amener à mieux utiliser les ressources de la situation pour déséquilibrer son adversaire. Dans ces situations, il semble que ce choix fasse sens pour Jeanne et lui permette de construire une connaissance liée au choix du placement en retour de service. 


\section{Discussion}

Les résultats de cette étude de cas permettent de mettre en évidence la façon dont les situations mises en place par l'enseignant ainsi que les interactions sous forme de questionnements, permettent de transformer l'activité de l'élève. Les différents artefacts matériels (lignes du terrain, plots) et symboliques (règles de position du serveur, du receveur) utilisés par l'enseignant permettent à l'élève de transformer sa dynamique d'engagement, ses repères perceptifs et les connaissances qu'elle construit et mobilise pour agir. Cette transformation rentre en cohérence avec les apprentissages visés par l'enseignant de rechercher le déséquilibre en utilisant les ressources de l'environnement et notamment les différentes règles mises en place. Nous discuterons de ces résultats au regard de trois éléments : a) la familiarité des situations dans lesquelles les connaissances se construisent et s'actualisent pour constituer un agir compétent ; b) les conditions pour que les artefacts soient des éléments qui favorisent la construction de compétences dans d'autres situations ; c) les interactions enseignant-élève valorisant un questionnement des élèves.

\subsection{La familiarité des situations}

Une des caractéristiques centrales de la compétence concerne son actualisation dans des situations qui présentent une structure commune (Jonnaert \& al., 2009 ; Masciotra \& Medzo, 2009 ; Meirieu, 2005). Seulement, peu d'études empiriques se sont intéressées à définir cette structure commune du point de vue des acteurs, c'est à dire ce qui permet aux individus de pouvoir identifier et mobiliser les connaissances nécessaires pour y répondre. La structure commune des situations étudiées, du point de vue de l'enseignant, possède plusieurs caractéristiques: a) des matchs en un contre un ; b) gérés au temps par l'enseignant ; c) en montantes-descendantes après avoir joué des finales sur le terrain. Cette structure varie autour de plusieurs paramètres : a) la position du serveur et/ou du réceptionneur; b) des handicaps ou des avantages pour l'un des deux joueurs. Nos résultats mettent en évidence que la transformation de l'activité de Jeanne se caractérise par une orientation de son engagement vers la recherche du déséquilibre et le développement de plusieurs connaissances liées aux moyens à utiliser pour déséquilibrer son adversaire. De plus, nous avons observé une transformation des repères pris par Jeanne (perceptifs sur le terrain et sur son adversaire, ou encore mnémoniques sur les consignes de l'enseignant) qui contribuent à la construction et à la transformation de ses 


\section{eJRIEPS spécial 2 (juillet 2019)}

connaissances pour agir. Nous pouvons faire l'hypothèse que la transformation de l'activité de l'élève concernant le renvoi du volant pour provoquer le déséquilibre peut s'expliquer par la stabilité des situations utilisées par l'enseignant entre les deux séances étudiées. Lors de la Séance 2, Jeanne est plutôt engagée dans l'application et la compréhension des consignes, et construit des connaissances liées à ces consignes. C'est lors de la Séance 3, dans des situations similaires à la séance précédente, qu'elle transforme son engagement vers la recherche du déséquilibre et construit plusieurs connaissances liées à l'utilisation des ressources de la situation pour déséquilibrer son adversaire. Nous pouvons considérer que cette structure commune est un moyen de ritualiser l'activité des élèves (Adé, 2010), pour les aider à transformer leur activité au sein de ces situations similaires. Aider les élèves à repérer les éléments familiers dans les situations proposées par l'enseignant semble donc une piste professionnelle à envisager pour contribuer au développement des compétences.

\subsection{L'utilisation d'artefacts pour transformer l'activité des élèves}

L'utilisation d'artefacts symboliques et matériels dans l'enseignement et la formation fait consensus dans la littérature pour aider à transformer l'activité des apprenants, à travers notamment l'amplification des conditions dans lesquelles l'expérience se construit (Adé, 2010 ; Hutchins, 1995 ; Sève \& Terré, 2017). Les artefacts cognitifs sont des offres disponibles dans l'environnement, qui amplifient les actions des apprenants selon leurs intentions (Norman, 1993). Nos résultats montrent que les différentes composantes de l'activité de Jeanne se transforment en lien avec l'utilisation des différents artefacts proposés dans les situations par l'enseignant (règles du jeu, plots et lignes). Ils peuvent apparaitre d'abord comme une contrainte pour Jeanne : elle cherche à appliquer les règles et à les comprendre ; puis ils deviennent ensuite des ressources pour agir : Jeanne les utilise au service du déséquilibre de son adversaire. Ces différentes règles font sens pour Jeanne, et peuvent alors être considérées comme des artefacts qui viennent favoriser la construction de connaissances lors de l'action en situation (Durand, 2008). En effet, la signification fonctionnelle des artefacts utilisés par l'enseignant n'est pas d'emblée donnée : leur utilisabilité est personnelle à chaque acteur, pour qui ils deviennent significatifs à un moment donné de leur apprentissage en fonction des engagements qui les animent en situation (Adé et al., 2010). La situation doit donc être signifiante et mobilisatrice pour l'élève, seule condition pour qu'elle soit gage d'apprentissage (Perrenoud, 1995 ; Sève \& Terré, 2017). Une question reste cependant en suspens concernant les conditions dans lesquelles les artefacts contribuent à développer un agir compétent, au-delà des situations dans 


\section{eJRIEPS spécial 2 (juillet 2019)}

lesquelles ces artefacts ont été utilisés. En effet, la similarité entre le contexte d'apprentissage et celui de mobilisation semble être un levier essentiel au développement des compétences (Terré, Sève \& Saury, 2016). Mais les caractéristiques situées et contextuelles de la compétence peuvent aussi être un frein à son actualisation dans des situations plus larges, voire extra-scolaires (Lemaitre \& Hatano, 2007 ; Van der Maren \& Loye, 2011). Dès lors, il semble intéressant de pister les conditions dans lesquelles les artefacts utilisés dans les situations sont susceptibles d'aider les élèves à actualiser leurs connaissances dans d'autres situations similaires mais qui sont dénuées de ces artefacts. Une analyse plus longue de l'activité d'élèves permettrait de rendre compte des processus de typification des connaissances au sein de situations familières.

\subsection{Les interactions enseignant-élève sous forme de questionnements}

Nos résultats mettent en évidence un moment d'interaction entre l'enseignant et Jeanne qui lui permet d'invalider une connaissance et d'en construire une autre lui permettant d'agir plus efficacement dans la situation (Tableau III). L'enseignant cherche toujours à questionner les élèves sur ce qu'ils font, pour les faire réfléchir sans forcément leur donner la solution. Cette modalité de questionnement a pour objectif d'orienter les élèves dans leur réflexion et dans leur construction du savoir enseigné (Maulini, 2005), mais aussi d'accéder au « monde propre » de l'élève en le questionnant sur ses préoccupations, ce qu'il cherche à faire pour pouvoir mieux le guider (Sève, 2014). Notre étude de cas montre que pour Jeanne, ce questionnement fonctionne et lui permet de construire une connaissance liée au placement en position de receveur qui lui permet d'être moins en retard que lorsqu'elle partait de la ligne du fond. Mais l'étude de l'activité de l'enseignant révèle qu'avec d'autres élèves, cette modalité d'interaction ne fait pas toujours sens. Se pose ici la question de savoir si ce mode d'interaction peut répondre aux besoins de tous les élèves. En effet, il semble qu'un aspect essentiel de l'approche par compétences concerne le fait de chercher à construire ses connaissances à partir de problèmes posés. Mais cette conception de l'enseignement des compétences à travers la compréhension et la construction de ses connaissances semble accentuer les inégalités entre les élèves, encore plus qu'un enseignement traditionnel (Chenu, Crahay \& Lafontaine, 2014 ; Hirtt, 2009 ; Morlaix, 2009). II semble donc intéressant de poursuivre nos travaux par l'étude d'élèves aux profils plus variés, et notamment ceux les plus en difficulté, même si la barrière du volontariat pour participer aux entretiens est parfois difficile à franchir pour ces élèves. 


\section{eJRIEPS spécial 2 (juillet 2019)}

\section{Conclusion}

À travers notre étude, nous avons pu voir que l'enseignement par compétences, tel que nous l'avons défini, amène une transformation de l'activité de l'élève pour agir. Face à des approches transmissives de savoirs décontextualisés, l'approche par compétences cherche plutôt à ce que les élèves construisent des connaissances typifiées (i.e., des types) à partir des différentes ressources proposées par l'enseignant. En analysant l'expérience de Jeanne, à travers ses différentes composantes, nous avons pu rendre compte de la transformation de son activité entre deux séances : du renvoi du volant à la recherche du déséquilibre de l'adversaire par l'utilisation des ressources de la situation. Plusieurs conditions semblent ressortir pour aider à développer un agir compétent en EPS : a) structurer le parcours de formation des élèves sur la base de situations d'apprentissage possédant des traits de familiarité et les aider à repérer ces traits de familiarité ; b) favoriser des situations problèmes où chaque élève peut construire des connaissances selon ses propres ressources et expériences; c) favoriser des interventions de l'enseignant sous forme de questionnements pour aider les élèves à construire des connaissances pour agir, à partir de leur expérience vécue et de ce qu’ils cherchent à faire.

\section{Bibliographie}

Adé, D. (2010). Les objets comme médiateurs dans l'activité professionnelle des enseignants débutants d'Education physique et sportive. In Adé, D., \& De SaintGeorges, I. (dir.), Les objets dans la formation. Usages, rôles et significations (pp. 77-95). Toulouse : Octarès.

Adé, D., Jourand, C., \& Sève, C. (2010). L'inscription contextuelle de l'activité en course en durée. Education \& didactique, 4(3), 7-22.

Adé, D., Picard, M., \& Saury, J. (2013). Les ressources exploitées par les élèves pour agir dans un format pédagogique en atelier: Une étude empirique lors de leçons de musculation en Education Physique et Sportive. eJRIEPS, 30, 26-50.

Bril, B. (2002). Apprentissage et contexte. Intellectica, 35(2), 251-268.

Chauvigné, C., \& Coulet, J. C. (2010). L'approche par compétences : un nouveau paradigme pour la pédagogie universitaire ?. Revue française de pédagogie, 172, 15-28.

Chenu, F., Crahay, M., \& Lafontaine, D. (2014). Par-delà l'approche par compétences : quelle place réserver aux savoirs, à leur enseignement et à leur évaluation ?. Education \& Formation, e302, 17-29. 


\section{eJRIEPS spécial 2 (juillet 2019)}

Cortesero, R. (2013). La notion de compétences : clarifier le concept, en mesurer les enjeux. Bulletin d'études et de synthèses de l'Observatoire de la jeunesse, 12, 1-4.

Delignières, D. (2009). Complexité et compétences. Paris : Revue EP\&S.

Develay, M. (2015). D'un programme de connaissances à un curriculum de compétences.

Bruxelles : De Boeck Supérieur.

Dubois, D., \& Poitou, J. (2002). Des «normes catégorielles » : structuration cognitive et/ou linguistique des catégories sémantiques. Intellectica, 2(35), 217-249.

Durand, M. (2007). Situations de l'action, dispositions à agir et trajectoires d'activité chez des enseignants débutants et des formateurs de terrain. Formation et pratiques d'enseignement en questions, 6, 59-74.

Durand, M. (2008). Un programme de recherche technologique en formation des adultes. Éducation \& didactique, 2(3), 97-121.

Epinoux, N. (2014). Les compétences sociales et l'apprentissage coopératif au collège : enjeux et perspectives. Apprendre à coopérer pour réaliser un projet collectif en EPS et en Sciences Physiques. Thèse de doctorat non publiée, STAPS, soutenue le 12 décembre 2014 à l'Université de Bordeaux.

Evin, A., Sève, C., \& Saury, J. (2013). Activité de l'enseignant et dynamique coopérative au sein de dyades d'élèves. Une étude de cas dans des tâches d'escalade en Education Physique. Recherches en Education, 15, 109-119.

Gal-Petitfaux, N., \& Durand, M. (2001). L'enseignement de l'éducation physique comme « action située » : propositions pour une approche d'anthropologie cognitive. STAPS, 55(2), 79-100.

Gal-Petiffaux, N., \& Ria, L. (2004). Apprentissage par l'action et construction d'indices de typicalité : le cas des enseignants débutants en éducation physique et sportive. In Loquet, M., \& Léziart, Y. (dir). Cultures sportives et artistiques. Formalisation des Savoirs Professionnels, pp. 217-220, Rennes : Presses Universitaires de Rennes.

Gendron, M., Royer, É., Bertrand, R., \& Potvin, P. (2005). Les troubles du comportement, la compétence sociale et la pratique d'activités physiques chez les adolescents. Revue des sciences de l'éducation, 31(1), 211-233.

Gréhaigne, J.F, Poggi, M.P., \& Zerai, Z. (2017). L'enseignement et l'apprentissage des connaissances et des compétences motrices utiles en sport collectif. eJRIEPS, 40, 163-184. 


\section{eJRIEPS spécial 2 (juillet 2019)}

Gresham, F. M., Van, M. B., \& Cook, C. R. (2006). Social skills training for teaching replacement behaviors: Remediating acquisition deficits in at-risk students. Behavioral Disorders, 31(4), 363-377.

Guérin, J. (2011). Activité collective et apprentissage : contribution à un programme de recherche en ergonomie cognitive sur le terrain de l'enseignement et de la formation. Habilitation à diriger des recherches, soutenue le 25 novembre 2011 à l'Université de Bretagne occidentale Brest.

Hirtt, N. (2009). L’approche par compétences : une mystification pédagogique. L'école démocratique, 39, 1-34.

Hutchins, E. (1995). Cognition in the Wild. Cambridge: MIT Press.

Jonnaert, P., Barrette, J., Boufrahi, S., \& Masciotra, D. (2004). Contribution critique au développement des programmes d'études : compétences, constructivisme et interdisciplinarité. Revue des sciences de l'éducation, 30(3), 667-696.

Jonnaert, P., Ettayebi, M., \& Defise, R. (2009). Curriculum et compétences : un cadre opérationnel. Bruxelles : De Boeck Supérieur.

Lemaître, D., \& Hatano, M. (2007). Usages de la notion de compétence en éducation et en formation. Paris : L'Harmattan.

Masciotra, D., \& Medzo, F. (2009). Développer un agir compétent : Vers un curriculum pour la vie. Louvain-la-Neuve, Bruxelles : De Boeck Supérieur.

Masciotra, D., Morel, D., \& Matthieu (2011). Apprendre par l'expérience active et située. La méthode ASCAR. Québec : Presses de I'Université du Québec.

Maulini, O. (2005). Questionner pour enseigner et pour apprendre. Le rapport au savoir dans la classe. Issy-les-Moulineaux : ESF.

Meirieu, P. (2005). Si la compétence n'existait pas, il faudrait l'inventer. IUFM de Paris Collège des CPE. Disponible à l'adresse : https://meirieu.com/ARTICLES/SUR\%20LES\%20COMPETENCES.pdf

Mons, N., Duru-Bellat, M., \& Savina, Y. (2012). Modèles éducatifs et attitudes des jeunes : une exploration comparative internationale. Revue française de sociologie, 53(4), 589-622.

Morlaix, S. (2009). Compétences des élèves et dynamique des apprentissages. Rennes : Presses Universitaires de Rennes.

Nagels, M. (2011). Un modèle empirique de l'évaluation régulatrice du développement des compétences. Actes du $23 e$ colloque de l'ADMEE-Europe. Evaluation et enseignement supérieur (pp. 203-212). Université Paris Descartes, France. 


\section{eJRIEPS spécial 2 (juillet 2019)}

Norman, D. A. (1993). Cognition in the head and in the world: An introduction to the special issue on situated action. Cognitive Science, 17(1), 1-6.

Perrenoud, P. (1995). La fabrication de l'excellence scolaire : du curriculum aux pratiques d'évaluation. Genève : Droz.

Perrenoud, P. (2011). Quand l'école prétend préparer à la vie... Des compétences ou d'autres savoirs. Paris : ESF.

Poizat, G., Salini, D., \& Durand, M. (2013). Approche énactive de l'activité humaine, simplexité et conception de formations professionnelles. Education Sciences \& Society, 4(1).

Rey, B. (1996). Les compétences transversales en question. Paris : ESF.

Rey, B. (2014). Compétence et évaluation en milieu scolaire : une relation complexe. In Dierendonck, C., Loarer, E., \& Rey, B. (dir), L'évaluation des compétences en milieu scolaire et en milieu professionnel (pp. 8-30). Bruxelles : De Boeck Supérieur.

Rey, O. (2012). Le défi de l'évaluation des compétences. Dossier d'actualité Veille et Analyse, $\mathrm{n}^{\circ} 76$, juin. Lyon : ENS de Lyon.

Richard, J. F. (1990). Les activités mentales. Comprendre, raisonner, trouver des solutions. Paris : Armand Colin.

Roegiers, X. (2017). De la connaissance à la compétence. Evaluer le potentiel d'action par un QCM. Recherche fondamentale inédite. Bruxelles : Peter Lang.

Rosch, E. (1978). Principles of categorization. In Rosch, E., \& Lloyd, BB. (dir). Cognition and Categorization, 27-48, Hillsdale, NJ : Lauwrence Erlbaum Associates.

Rosch, E., \& Mervis, C. B. (1975). Family resemblances: Studies in the internal structure of categories. Cognitive psychology, 7(4), 573-605.

Saury, J., Adé, D., Gal-Petitfaux, N., Huet, B., Sève, C., \& Trohel, J. (2013). Actions, significations et apprentissages en EPS. Une approche centrée sur les cours d'expériences des élèves et des enseignants. Paris : Revue EP\&S.

Sève, C. (2014). Le suivi. Entre guidage, accompagnement et enquête. E-nov eps (6).

Sève, C., \& Gal-Petitfaux, N. (2015). Les techniques corporelles en éducation physique. In Durand, M, Hauw, D., \& Poizat, G. (dir). L'apprentissage des techniques corporelles (pp. 101-114). Paris : PUF.

Sève, C., \& Leblanc, S. (2003). Exploration et exécution en situation : singularité des actions, construction de types et apprentissage dans deux contextes différents. Recherche \& Formation, 42(1), 63-74. 


\section{eJRIEPS spécial 2 (juillet 2019)}

Sève, C., \& Terré, N. (2017). L'EPS du dedans. Pour un enseignement inclusif, citoyen et ouvert vers le futur. Paris : Revue EP\&S.

Suchman, L. (1987). Plans and situated actions: the problem of human/machine communication. Cambridge : Cambridge University Press.

Terré, N. (2015). Les connaissances des élèves en Education physique : étude de la dynamique de construction et d'actualisation des connaissances à partir de l'expérience de lycéens à l'échelle d'un cycle d'apprentissage en escalade. Thèse de doctorat non publiée en STAPS, Université de Nantes.

Terré, N., Sève, C., \& Saury, J. (2016). Une approche énactive du développement des compétences en milieu scolaire. Éducation et francophonie, 44(2), 68-85.

Theureau, J. (1992). Le cours d'action, analyse sémio-logique : essai d'une anthropologie cognitive située. Bern : Peter Lang.

Theureau, J. (2004). Le cours d'action : Méthode élémentaire. Toulouse : Octarès.

Theureau, J. (2006). Le cours d'action : Méthode développée. Toulouse : Octarès.

Van der Maren, J. M., \& Loye, N. (2011). A propos de quelques difficultés de l'évaluation des compétences. Réflexions critiques. Education Sciences \& Society, 2, 40 - 53.

Varela, F. (1989). Autonomie et Connaissance. Essai sur le vivant. Paris : Seuil.

\section{Textes institutionnels}

Décret n²006-830 du 11 juillet 2006 relatif au Socle Commun de Connaissances et de Compétences, publié au Journal Officiel du 12 juillet 2006.

Décret n²015-371 du 31 mars 2015 relatif au Socle Commun de Connaissances, de Compétences et de Culture, publié au Journal Officiel du 2 avril 2015.

Loi d'Orientation et de Programmation pour la Refondation de l'Ecole de la République. Loi n²013-595 du 8 juillet 2013.

Ministère de l'Education Nationale, de l'Enseignement Supérieur et de la Recherche (2016). Collège: mieux apprendre pour mieux réussir. Disponible à l'adresse : http://cache.media.education.gouv.fr/file/03_-_mars/62/2/College-Mieuxapprendre-pour-mieux-reussir_398622.pdf

Thélot, C. (2004). Pour la réussite de tous les élèves : rapport de la Commission du débat national sur l'avenir de l'Ecole présidée par Claude Thélot. Collection des rapports officiels : La documentation française 